\title{
An Equivalent Circuit Model for Transmitting Capacitive Micromachined Ultrasonic Transducers in Collapse Mode
}

\author{
Selim Olcum, Member, IEEE, F. Yalcin Yamaner, Student Member, IEEE, \\ Ayhan Bozkurt, Member, IEEE, Hayrettin Köymen, Senior Member, IEEE, \\ and Abdullah Atalar, Fellow, IEEE
}

\begin{abstract}
The collapse mode of operation of capacitive micromachined ultrasonic transducers (CMUTs) was shown to be a very effective way to achieve high output pressures. However, no accurate analytical or equivalent circuit model exists for understanding the mechanics and limits of the collapse mode. In this work, we develop an equivalent nonlinear electrical circuit that can accurately simulate the mechanical behavior of a CMUT with given dimensions and mechanical parameters under any large or small signal electrical excitation, including the collapse mode. The static and dynamic deflections of a plate predicted from the model are compared with finite element simulations. The equivalent circuit model can estimate the static deflection and transient behavior of a CMUT plate to within $5 \%$ accuracy. The circuit model is in good agreement with experimental results of pulse excitation applied to fabricated CMUTs. The model is suitable as a powerful design and optimization tool for collapsed and uncollapsed CMUTs.
\end{abstract}

\section{INTRODUCTION}

$\mathrm{C}$ APACITIVE micromachined ultrasonic transducers $\mathcal{U}$ (CMUTs) were introduced [1] as micromachined suspended plate structures with a moving top electrode and a rigid substrate electrode. When immersed in a liquid medium, CMUTs are capable of generating wideband acoustical pulses with more than $100 \%$ fractional bandwidth [2]. However, many applications require high transmitted pressures for increased penetration and signal quality. The power output capability of CMUTs can be increased by utilizing the collapsed state of the plates [3]-[5].

Accurate and fast simulation methods are necessary for understanding CMUT dynamics and for designing high-performance CMUTs. CMUT models are based either on finite element method (FEM) models [6], [7] or on equivalent circuits [8]-[11]. FEM simulations require long

Manuscript received November 22, 2010; accepted April 20, 2011. This work was supported by The Scientific and Technological Research Council of Turkey (TUBITAK) under project grants 107T921 and 110E216. S. Olcum gratefully acknowledges the financial support of TUBITAK and ASELSAN for their National Scholarship and Ph.D. Fellowship Programs. A. Atalar acknowledges the support of The Turkish Academy of Sciences (TUBA).

S. Olcum, H. Köymen, and A. Atalar are with Bilkent University, Electrical and Electronics Engineering Department, Ankara, Turkey (email: selim@ee.bilkent.edu.tr).

F. Y. Yamaner and A. Bozkurt are with Sabanci University, Electronics Engineering Department, Istanbul, Turkey.

Digital Object Identifier 10.1109/TUFFC.2011.1966 simulation times for accurate results. This makes them relatively inconvenient for design and optimization purposes. On the other hand, parametric electrical equivalent circuits [10]-[12] based on Mason's model [13] are more suitable for design purposes. In recent years, the efforts to model the nonlinear behavior of CMUTs under large excitations [8], [14], [15] have increased. However, all of those models are valid only for the uncollapsed state. The collapse mode of a CMUT plate has been explored only with experiments [3]-[5] and simulations performed by FEM [5], [16], [17]. CMUTs in the collapsed state lack fast and accurate equivalent circuit models.

In this work, we develop an electrical equivalent circuit model that can be used to simulate the mechanical behavior of a transmitting CMUT under any electrical excitation. The model is dependent on plate dimensions and mechanical properties and it can predict the plate movement in the collapsed state as well as in the uncollapsed state. The simulation results of the circuit for static and dynamic deflections are compared with electrostatic and transient FEM simulation results. Acoustical pressure generated at the surface of a CMUT plate under a large pulse excitation is calculated by the circuit model and compared with transient FEM simulation and experimental results performed on fabricated CMUTs.

\section{Static Deflection of a CMUT}

CMUTs usually have two electrodes: a top electrode in the plate and a bottom electrode in the substrate. As an electric potential is applied between the top electrode and the bottom electrode, the plate deflects because of the electrostatic forces, resulting in a transmission of a pressure wave to the immersion medium. It has been demonstrated in previous studies [3], [4] that the collapse mode drastically increases the pressure amplitude. More recently, it has been shown [5] that for the highest pressure generation, full electrode coverage is necessary. Using a full electrode, more force can be generated than the force generated by partial electrode coverage for the same applied voltage. In this paper, we assume that the electrodes have full coverage over the plate region. A cross-sectional view of a collapsed plate is illustrated in Fig. 1, establishing our notation.

We use the rms displacement, $x_{\mathrm{rms}}(t)$, 


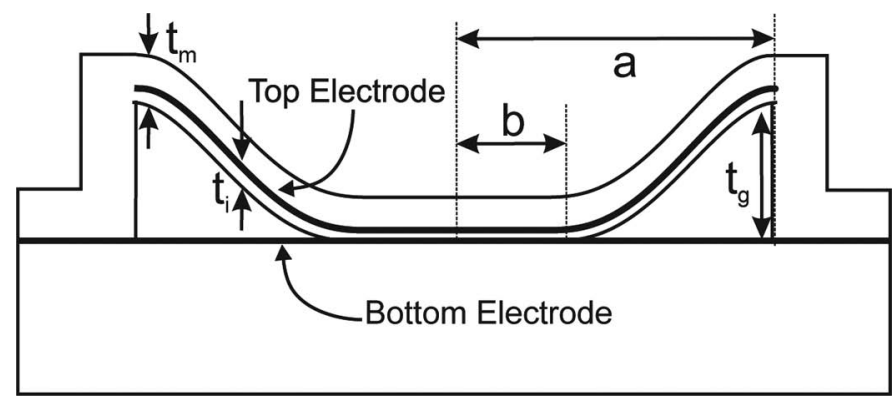

Fig. 1. Cross-sectional view of a collapsed clamped circular plate with radius $a$, thickness $t_{m}$, and gap height of $t_{g}$. The top electrode is at a distance $t_{i}$ above from the gap. $b$ is the contact radius. The bottom electrode is assumed to be at the top of the substrate.

$$
x_{\mathrm{rms}}(t) \equiv \sqrt{\frac{1}{\pi a^{2}} \int_{0}^{a} 2 \pi r x^{2}(r, t) \mathrm{d} r},
$$

rather than the average displacement, $x_{\text {avg, }}$,

$$
x_{\mathrm{avg}}(t) \equiv \frac{1}{\pi a^{2}} \int_{0}^{a} 2 \pi r x(r, t) \mathrm{d} r,
$$

of the plate as the lumped displacement measure, where $x(r, t)$ signifies the plate displacement as a function of radial distance and time. The choice of $x_{\mathrm{rms}}$ as the lumped displacement measure requires a correction on the definition of a lumped force acting on the plate. The correction is performed by dividing the total force integrated over the plate by a profile-dependent factor, $\kappa$. A justification of this correction factor is given in Appendix A. For the uncollapsed state, the value of $\kappa$ was calculated in $[15]^{1}$ as $3 / \sqrt{5}$. In the collapsed ${ }^{2}$ state, the deflection profile changes as a function of $x_{\text {rms }}$, hence the value of $\kappa$ depends on $x_{\text {rms: }}$ :

$$
\kappa=\frac{x_{\mathrm{rms}}}{x_{\mathrm{avg}}}= \begin{cases}\frac{3}{\sqrt{5}} & \text { for } x_{\mathrm{rms}} \leq \frac{t_{g}}{\sqrt{5}} \\ \frac{3}{\sqrt{5}}-\gamma_{0}\left(x_{\mathrm{rms}}-\frac{t_{g}}{\sqrt{5}}\right) & \text { for } x_{\mathrm{rms}}>\frac{t_{g}}{\sqrt{5}}\end{cases}
$$

where $\gamma_{0}$ is a coefficient that depends on plate dimensions.

The deflection of a circular clamped plate under uniform pressure can be calculated using the equations given by Timoshenko [18] and for the boundary conditions given in [5] for both uncollapsed and collapsed states. For convenience, these equations are repeated in Appendix B. The given equations are valid as long as the forces acting on the plate are uniformly distributed over the surface and the amount of deflection does not cause stress stiffening of the CMUT plate (i.e., deflection-to-thickness ratio less than 20\%) [19], [20]. Because the separation of the electrodes is not constant throughout the cavity, the electrostatic force on a CMUT plate is not uniformly distributed,

\footnotetext{
${ }^{1}$ In [15], the operation for correcting the forces with respect to $\mathrm{rms}$ state variables is missing and should be included for the correct results.

$2 \mathrm{~A}$ clamped circular plate under uniform pressure touches the substrate when $x_{\text {avg }}=t_{g} / 3$ or $x_{\mathrm{rms}}=t_{g} / \sqrt{ } 5$.
}

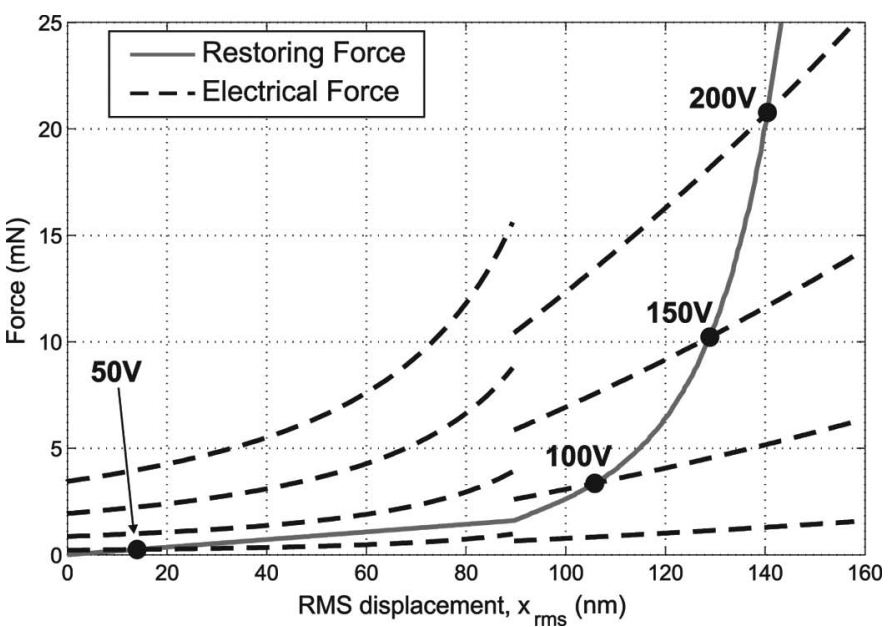

Fig. 2. Mechanical restoring force (solid line) and electrostatic attraction forces (dashed lines) when the bias is $50,100,150$, and $200 \mathrm{~V}$ as a function of $x_{\mathrm{rms}}$. The dimensions of the silicon nitride plate: $a=25 \mu \mathrm{m}, t_{m}$ $=1.5 \mu \mathrm{m}, t_{i}=0.4 \mu \mathrm{m}$, and $t_{g}=0.2 \mu \mathrm{m}$. The intersection point of the curves is the static equilibrium position when the atmospheric pressure is neglected.

making the derivation of an analytical expression for plate deflection very difficult, if not impossible.

At this point, we make the assumption that the deflection profile caused by the nonuniform attraction force of an applied voltage can be approximated by the deflection caused by a uniform pressure, $P$ with the same total force. We will show that this is a good assumption even in the collapse mode when the attraction force is highly nonuniform. Because the force caused by the uniform pressure is balanced by the mechanical restoring force of the plate, we can express the lumped restoring force $F_{\mathrm{r}}$ in terms of $P$ by including the correction factor $\kappa$ as

$$
F_{\mathrm{r}}=\frac{1}{\kappa} P \pi a^{2}
$$

With a given applied pressure $P$, the deflection profile, and hence $x_{\mathrm{rms}}$, can be estimated [5]. $F_{\mathrm{r}}$ is plotted in Fig. 2 as a function of $x_{\text {rms. }}$. It is a linear function in the uncollapsed region, but it becomes highly nonlinear in the collapsed region.

The lumped electrical attraction force, $F_{\mathrm{e}}$, generated by an applied voltage, $V_{\text {in }}$, can be determined in terms of $x_{\mathrm{rms}}$ by using the formulations in [14] and [15] as follows:

$$
F_{\mathrm{e}}\left(V_{\mathrm{in}}, x_{\mathrm{rms}}\right)=\frac{1}{2 \kappa} \frac{\mathrm{d} C}{\mathrm{~d} x_{\mathrm{rms}}} V_{\mathrm{in}}^{2}
$$

where $C$ is the capacitance between the two electrodes as a function of $x_{\mathrm{rms}} . F_{\mathrm{e}}$ is not only nonlinear, but is also discontinuous at the point where the plate touches the substrate, because there is a sudden change in the derivative of $C$ at that point. The calculated $F_{\mathrm{e}}\left(x_{\mathrm{rms}}\right)$ for different voltages are also plotted in Fig. 2 as dashed curves.

Although a closed form expression of deflection as a function of applied voltage cannot be determined, a nu- 


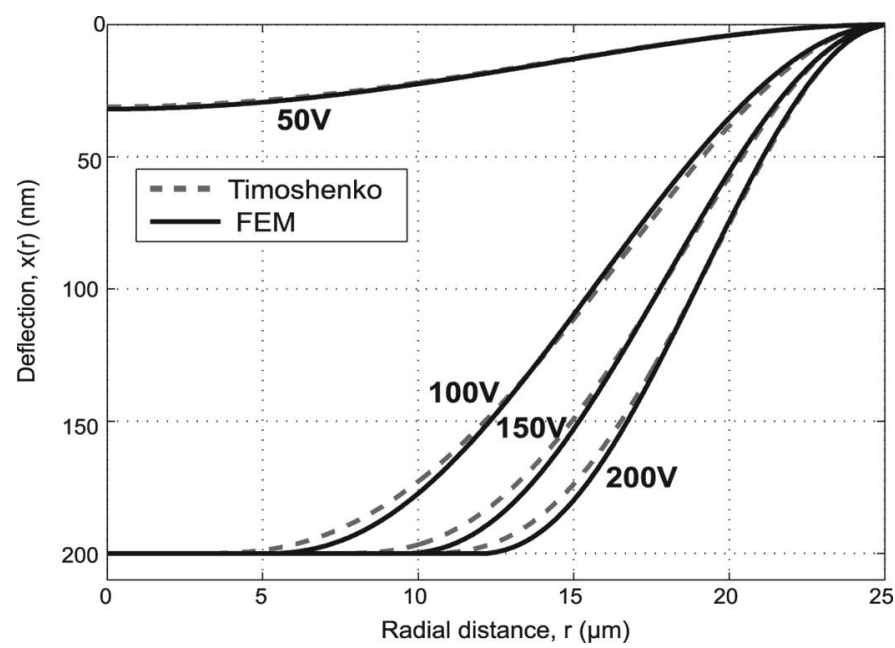

Fig. 3. Displacement profiles calculated by electrostatic FEM simulations (solid lines) and by Timoshenko's plate deflection under uniform pressure (dashed lines) for a silicon nitride plate with $a=25 \mu \mathrm{m}, t_{m}=$ $1.5 \mu \mathrm{m}, t_{i}=0.4 \mu \mathrm{m}$, and $t_{g}=0.2 \mu \mathrm{m}$ for different values of bias. The atmospheric pressure is neglected.

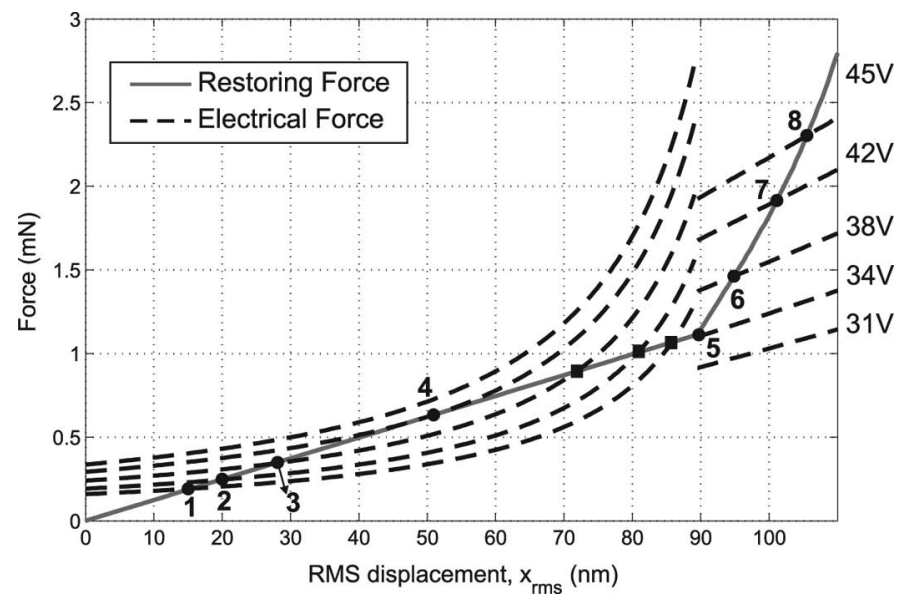

Fig. 4. The electrostatic attraction and mechanical restoring forces of a silicon nitride plate with $a=30 \mu \mathrm{m}, t_{m}=1.5 \mu \mathrm{m}, t_{i}=0.2 \mu \mathrm{m}, t_{g}=$ $0.2 \mu \mathrm{m}$ for different values of dc bias as a function of $x_{\mathrm{rms}}$. The atmospheric pressure is neglected.

merical solution is possible by determining the intersection of the nonlinear electrostatic attraction and mechanical restoring force curves. The intersection points in Fig. 2 give the static equilibrium displacement points. In Fig. 3 , the profiles calculated by the proposed method are compared with the profiles obtained by electrostatic FEM simulations for different bias values; the good agreement justifies the assumption we made previously. We note that the effect of atmospheric pressure can be included by adding a constant force term on top of the electrostatic attraction force.

We show the restoring and attraction forces around the collapse point in more detail in Fig. 4 for a CMUT with different dimensions. As the applied voltage is increased from 31 to $45 \mathrm{~V}$, the static displacement follows the intersection points, $1,2,3,4$, and 8 . We note that at $42 \mathrm{~V}$, the electrical and restoring force curves are tangential to each

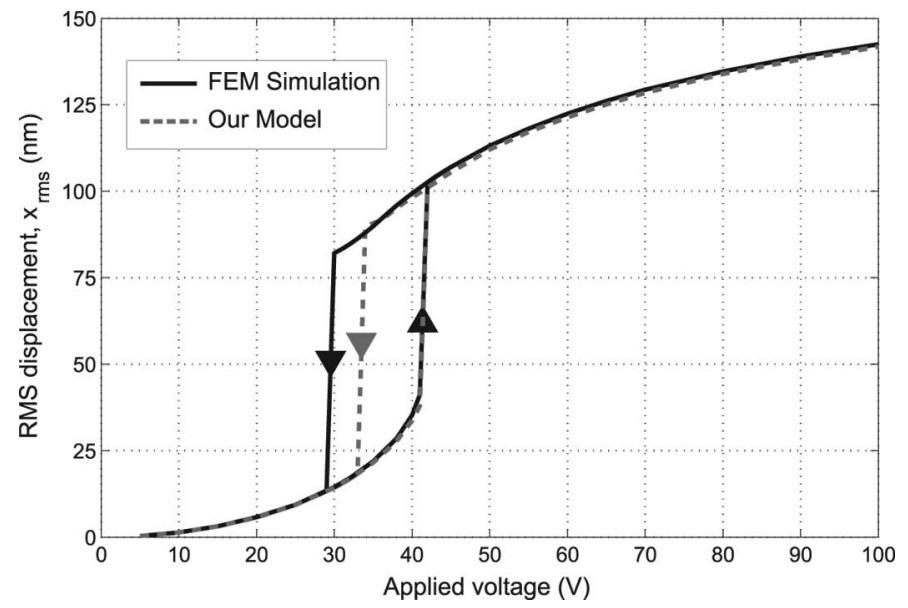

Fig. 5. RMS displacement of a silicon nitride plate with $a=30 \mu \mathrm{m}, t_{m}$ $=1.5 \mu \mathrm{m}, t_{i}=0.2 \mu \mathrm{m}, t_{g}=0.2 \mu \mathrm{m}$ as a function of applied voltage. The displacements calculated by our method (dashed lines) are compared with FEM simulation results (solid lines). The effect of the atmospheric pressure is neglected.

other. The intersection points signified by squares are the unstable solutions of the plate displacement. On the other hand, if we reduce the voltage starting from 45 toward $31 \mathrm{~V}$, the static equilibrium displacement will follow the points, $8,7,6,5$, and 1 , demonstrating the well known hysteretic behavior of CMUTs. Our model predicts the collapse and snap-back voltages as 42 and $33 \mathrm{~V}$, whereas FEM simulations predict the same voltages as 42 and $29 \mathrm{~V}$, respectively. The difference in the value of the snapback voltage is attributed to the variation in the plate profile around the snap-back point, because we always use the profiles generated by a uniformly distributed load.

In Fig. 5, $x_{\mathrm{rms}}$ as a function of applied voltage is shown in comparison to FEM simulation results. The error between the rms deflections and the electrostatic FEM simulation results is less than $1 \%$ except for voltages in the close vicinity of the snap-back.

In the uncollapsed state, $F_{\mathrm{e}}\left(V_{\mathrm{in}}, x_{\mathrm{rms}}\right)$ is given in [14] and repeated in Appendix B-A for convenience. In the collapsed region, we fit a third-order polynomial to the nonlinear function and find coefficients $\alpha_{0}, \alpha_{1}, \alpha_{2}$, and $\alpha_{3}$. Hence, we can express $F_{\mathrm{e}}\left(V_{\mathrm{in}}, x_{\mathrm{rms}}\right)$ analytically as

$$
F_{\mathrm{e}}\left(V_{\mathrm{in}}, x_{\mathrm{rms}}\right)=\frac{1}{2 \kappa} V_{\mathrm{in}}^{2} \begin{cases}\text { Eq. }(18), & x_{\mathrm{rms}} \leq \frac{t_{g}}{\sqrt{5}} \\ \sum_{n=0}^{3} \alpha_{n} x_{\mathrm{rms}}^{n}, & x_{\mathrm{rms}} \geq \frac{t_{g}}{\sqrt{5}} .\end{cases}
$$

In the uncollapsed region, the restoring force of the plate, $F_{\mathrm{r}}\left(x_{\mathrm{rms}}\right)$, can be determined using (16) in Appendix $\mathrm{B}-\mathrm{A}$. Because of the highly nonlinear nature of the force in the collapsed region, a tenth-order polynomial with coefficients $\beta_{0}, \beta_{1}, \beta_{2}, \ldots, \beta_{10}$ is necessary for a good approximation. Hence, $F_{\mathrm{r}}\left(x_{\mathrm{rms}}\right)$ can be written as

$$
F_{\mathrm{r}}\left(x_{\mathrm{rms}}\right)=\frac{1}{\kappa} \begin{cases}\frac{64 \sqrt{5} \pi D}{a^{2}} x_{\mathrm{rms}}, & x_{\mathrm{rms}} \leq \frac{t_{g}}{\sqrt{5}} \\ \sum_{n=0}^{10} \beta_{n} x_{\mathrm{rms}}^{n}, & x_{\mathrm{rms}}>\frac{t_{g}}{\sqrt{5}} .\end{cases}
$$


TABle I. Equivalence Between Mechanical and Electrical Quantities.

\begin{tabular}{ll}
\hline Mechanical & $\begin{array}{l}\text { Equivalent } \\
\text { circuit }\end{array}$ \\
\hline$v_{\mathrm{rms}}$ & $i_{v}$ \\
$x_{\mathrm{rms}}$ & $q_{x}$ \\
$F_{\mathrm{atm}}$ & $V_{\mathrm{atm}}$ \\
$F_{\mathrm{e}}\left(V_{\mathrm{in}}, x_{\mathrm{rms}}\right)$ & $V_{\mathrm{e}}\left(V_{\mathrm{in}}, q_{x}\right)$ \\
$F_{\mathrm{r}}\left(x_{\mathrm{rms}}\right)$ & $V_{\mathrm{r}}\left(q_{x}\right)$ \\
$m$ & $L_{m}$ \\
$Z_{\mathrm{r}}$ & $Z_{\mathrm{r}}$ \\
\hline
\end{tabular}

The lumped force, $F_{\text {atm }}$, generated by the atmospheric pressure, $P_{0}$, is also a function of $x_{\mathrm{rms}}$, because the correction factor $\kappa$ is a function of $x_{\mathrm{rms}}$ :

$$
F_{\mathrm{atm}}\left(x_{\mathrm{rms}}\right)=\frac{1}{\kappa} P_{0} \pi a^{2}
$$

\section{Electrical Equivalent Circuit}

The dynamic problem can be solved by modeling the mass of the plate and the loading of the immersion medium as electrical circuit components similar to Mason's equivalent circuit model [10], [13]. The equivalence between mechanical variables and electrical variables can be established as detailed in Table I. Here, the velocity becomes current, the displacement becomes charge, and the forces are modeled by voltage sources. The equivalent circuit is shown in Fig. 6.

In this circuit, the mass, $m$, of the plate is represented by a series inductor, $L_{m}$ with a value equal to the mass of the plate $[15]$ :

$$
L_{m}=m=\rho t_{m} \pi a^{2},
$$

where $\rho$ is the density of the plate material.

The loading of the immersion medium can be modeled with a radiation impedance, $Z_{\mathrm{r}}$. $Z_{\mathrm{r}}$ of a circular CMUT plate for the uncollapsed mode was calculated previously [21]. It was shown ${ }^{3}$ that

$$
Z_{\mathrm{r}}=\pi a^{2} \rho_{0} c, \quad \text { for } a>\lambda,
$$

where $\lambda$ is the wavelength in the immersion medium and $\rho_{0}$ and $c$ are the density and the speed of sound in that medium, respectively. The condition of validity in (10) is not easily satisfied for typical CMUT cells. It was shown in [21] that for an array of cells with a total array radius, $A$, between $\lambda / 3$ and $\lambda$, the equivalent radiation impedance for the whole array is approximately half of the value given in (10):

$$
Z_{\mathrm{r}}=\frac{1}{2} \pi a^{2} \rho_{0} c, \quad \text { for } \lambda / 3<A<\lambda .
$$

\footnotetext{
${ }^{3}$ If we choose $v_{\text {avg }}$ rather than $v_{\text {rms }}$ as the through variable, the radiation impedance and inductance should be scaled by $\kappa^{2}$.
}

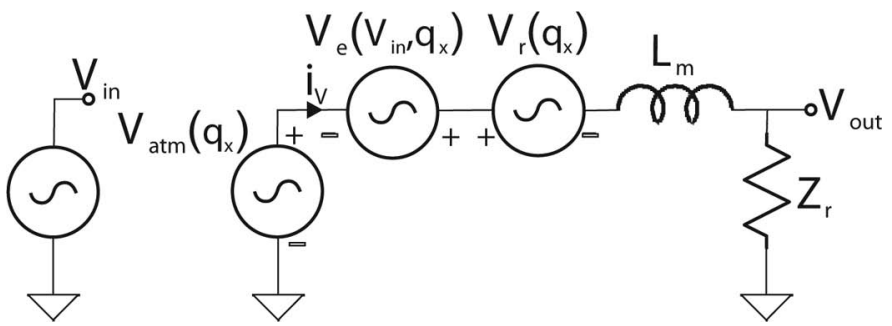

Fig. 6. Equivalent circuit model for simulating the behavior of CMUTs. $V_{\mathrm{r}}\left(q_{x}\right)$ stands for the series mechanical capacitance in Mason's equivalent circuit model. $V_{\mathrm{e}}\left(V_{\mathrm{in}}, q_{x}\right)$ represents the force generated at the mechanical side of the Mason's model. The shunt input capacitance of Mason's model is ignored, because we assume that CMUTs are driven by a voltage source.

TABLE II. Silicon Nitride Properties Used in Simulations.

\begin{tabular}{lc}
\hline Young's modulus, $E$ & $110 \mathrm{GPa}$ \\
Poisson's ratio, $\nu$ & 0.27 \\
Dielectric permittivity, $\varepsilon_{\mathrm{r}}$ & 5.4 \\
Density, $\rho$ & $3.1 \mathrm{~g} / \mathrm{cm}^{3}$ \\
\hline
\end{tabular}

We do not have the $Z_{\mathrm{r}}$ expression for the collapse mode CMUTs. In this work, we will assume that it can be approximated with $Z_{\mathrm{r}}$ of a piston ring transducer [22], corrected with the area ratio. Hence, we can use the expressions given previously.

\section{RESUlts}

We have tested the results of our equivalent circuit model by comparing them to FEM simulations and experimental results. A description of the FEM model utilized can be found in Appendix C. The material parameters used in the FEM and circuit model are given in Table II. The equivalent circuit is simulated using the LTSpice circuit simulator. ${ }^{4}$ The coefficients of the polynomials representing the nonlinear functions of the dependent voltage sources are input to LTSpice as parameters calculated externally by a parametric MATLAB code (The MathWorks, Natick, MA). ${ }^{5}$

\section{A. Comparison of the Equivalent Circuit Model to FEM}

For a demonstration of the large signal capability of the circuit model, we applied electrical pulses to a CMUT with amplitudes much greater than its collapse voltage. The resulting pressure amplitudes calculated by the equivalent circuit simulations are plotted in Fig. 7, along with the FEM simulation results. In the equivalent circuit, we use the radiation impedance expression given in (10), because in the FEM simulations we model the coupling medium

${ }^{4}$ LTSpice is public domain software and can be downloaded from http://www.linear.com/designtools/software (Linear Technology, Milpitas, CA).

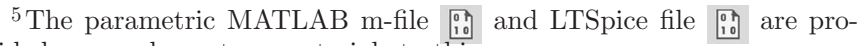
vided as supplementary materials to this paper. 


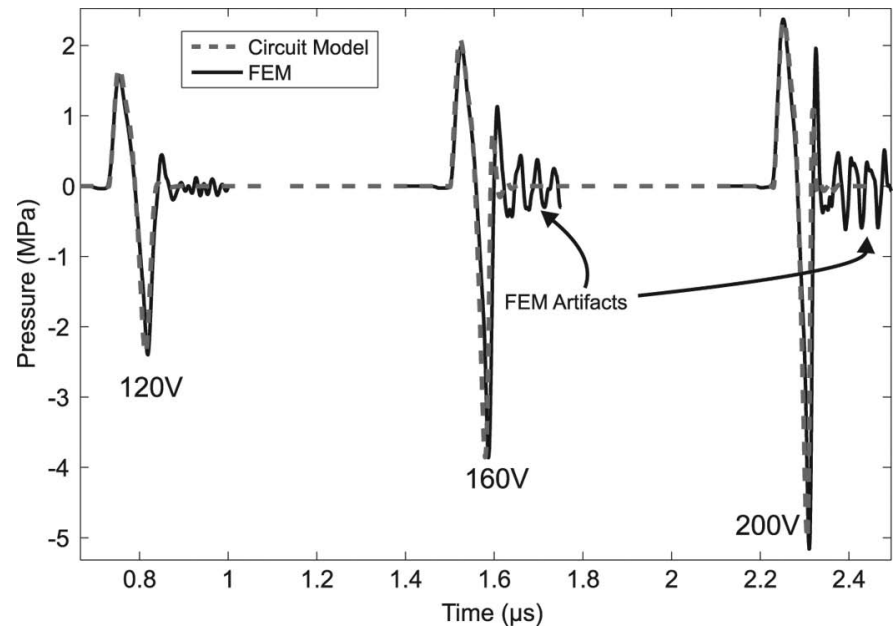

Fig. 7. Comparison of the calculated average pressure output using FEM (solid) and equivalent circuit (dashed) simulation results, when CMUTs are excited by 120,160 , and $200 \mathrm{~V}$ negative pulses on equal amplitude biases. The rise and fall times of the pulses are $20 \mathrm{~ns}$ and the pulse width is $40 \mathrm{~ns}$. The simulated CMUT has a silicon nitride plate with $a$ $=30 \mu \mathrm{m}, t_{m}=1.4 \mu \mathrm{m}, t_{i}=0.4 \mu \mathrm{m}, t_{g}=0.2 \mu \mathrm{m}$ resulting in a collapse voltage of $\sim 35 \mathrm{~V}$.

by a fluid column with rigid boundaries. This is a suitable model for a plate at the center of an infinite CMUT array, when there is no lateral displacement of the fluid.

The peak-to-peak amplitudes of the transmitted pressure waves are predicted within $3 \%$ and the pulse width of the prominent cycle is estimated within $5 \%$ accuracy in the worst case. During the pulse cycle, the plate is first released and then collapsed back to its initial position. Because the plate has a nonzero kinetic energy at the time it reaches its initial position, it oscillates at a high frequency for a while before it stabilizes. This behavior is predicted by the equivalent circuit model. The low-frequency oscillation seen in the FEM results, however, is a spurious oscillation as a result of rigidly defined stand regions and the lossless nature of the simulation.

The normalized transmission spectra of the CMUT for different excitations are shown in Fig. 8. The frequency spectra of the pressure waveforms calculated by the circuit model (dashed) and the FEM (solid) are in good agreement, especially at the low-frequency end. The error of the equivalent circuit simulation results in the lower-frequency end of the 3 - $\mathrm{dB}$ bandwidth is less than $5 \%$, whereas the error is less than $6 \%$ for the high-frequency end. The bandwidth and the center frequency of the generated acoustic pulse is also predicted by the equivalent circuit simulations within $6 \%$. Note that the error is smaller when the excitation amplitude is smaller. We believe that the error is due to the highly nonlinear nature of the collapsed CMUT.

The developed equivalent circuit model gives information about the plate movement as well. We can extract the information such as the contact radius, $b$, of a collapsed plate during a pulse cycle. The variation of $b$ as a function of time is plotted in Fig. 9, when the plate is excited with large electrical signals. As seen in the figure, the plate

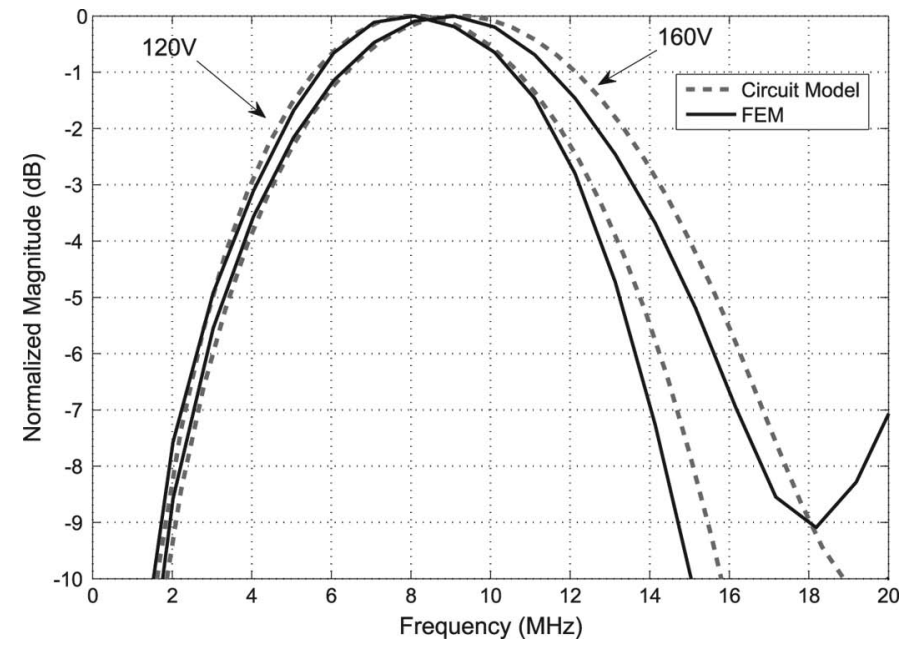

Fig. 8. Normalized spectra of the calculated acoustic pulses in Fig. 7.
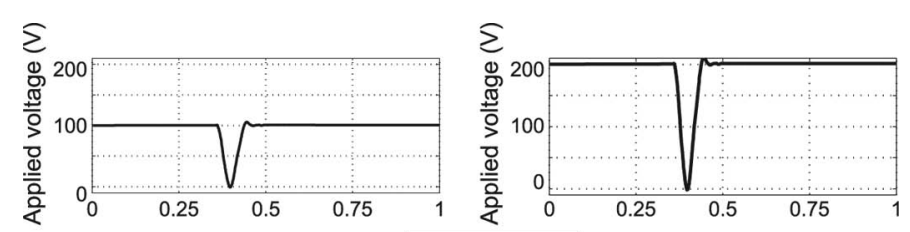

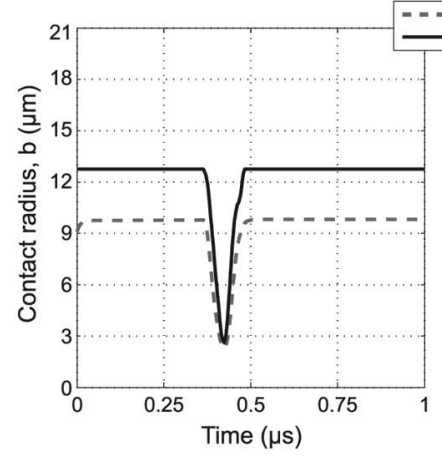

(a)

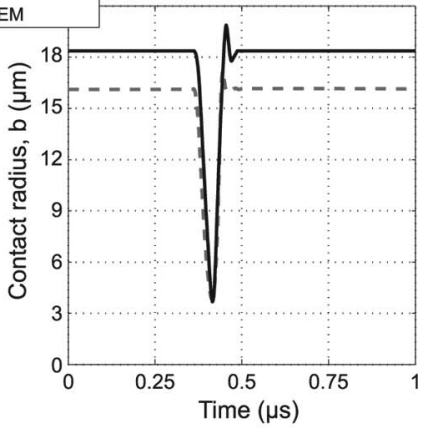

(b)
Fig. 9. Variation of the contact radius, $b$, as calculated by FEM (solid lines) and equivalent circuit simulation (dashed lines) as a function of time, when a CMUT is excited by negative (a) $100 \mathrm{~V}$ and (b) $200 \mathrm{~V}$ pulses on a bias with equal amplitude. The applied signals are depicted at the top. The simulated CMUT has a silicon nitride plate with $a=$ $30 \mu \mathrm{m}, t_{m}=1.4 \mu \mathrm{m}, t_{i}=0.4 \mu \mathrm{m}, t_{g}=0.2 \mu \mathrm{m}$.

does not lose contact with the substrate during the short pulse cycle, even though the input voltage is decreased to $0 \mathrm{~V}$. Even so, more than $7 \mathrm{MPa}$ peak-to-peak radiated pressure at the surface of the CMUT is possible using a $200 \mathrm{~V}$ pulse. If the duration of the pulse is increased, eventually the plate is released from the contact. The variation of the contact radius for pulses with different widths is shown in Fig. 10. The difference seen in the contact radii calculated by FEM and circuit model can be attributed to the assumed history-independent deflection profiles. Additionally, the circuit model is a lumped parameter simulator, whereas contact radius, $b$ is a distributed parameter heavily dependent on the deflection profile. The circuit model approximates all voltage-generated deflection profiles by uniform pressure-generated profiles, for which the contact radius is smaller (See Fig. 3). 

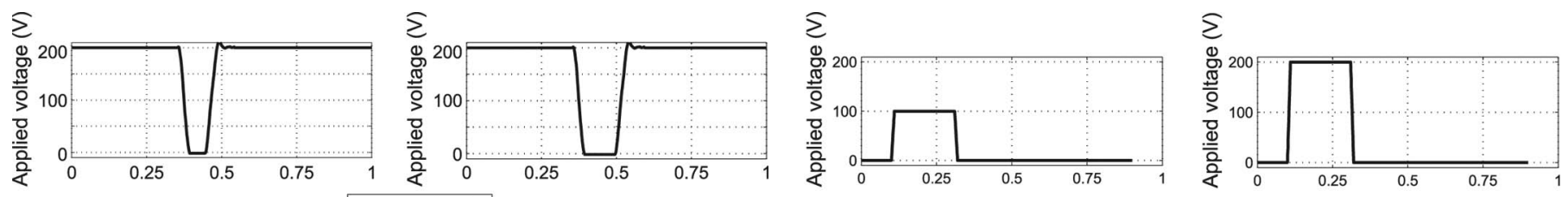

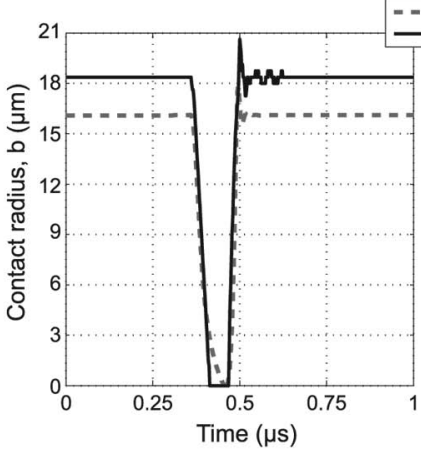

(a)

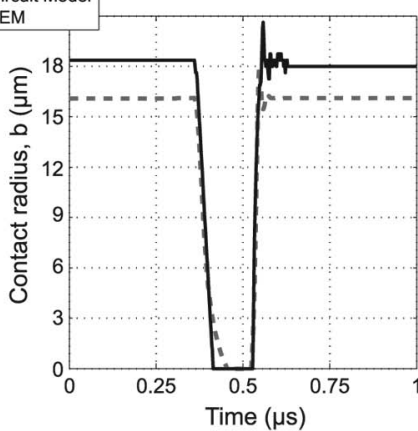

(b)

Fig. 10. Variation of the contact radius, $b$, as calculated by FEM (solid lines) and equivalent circuit simulation (dashed lines) as a function of time, when a CMUT is excited by $200 \mathrm{~V}$ pulses with different widths (a and b) on a bias with equal amplitude. The applied signals are depicted at the top. The simulated CMUT has a silicon nitride plate with $a=$ $30 \mu \mathrm{m}, t_{m}=1.4 \mu \mathrm{m}, t_{i}=0.4 \mu \mathrm{m}, t_{g}=0.2 \mu \mathrm{m}$.

As a further demonstration of the electrical model, we calculate the instantaneous displacement of a plate with small gap, collapsed because of the atmospheric pressure. Pulses of different amplitudes are applied to the CMUT. A rising voltage step collapses the plate further to the substrate, increasing $x_{\mathrm{rms}}$. The falling voltage step releases the plate to its initial position. The variation of the plate displacement is calculated by FEM simulations under the same electrical excitations and the results are depicted together in Fig. 11 for comparison. The oscillation of the plate seen in the FEM results, we believe, is an artifact of FEM as a result of rigidly defined stand regions and the lossless nature of the simulation. The vibrational wave generated on the plate by high-voltage excitation propagates to the stand regions and reflects back and oscillates on the plate. This behavior can be clearly identified in the transient FEM analysis ${ }^{6}$ of a deformed CMUT plate.

\section{B. Deep Collapsed Mode}

Using the developed model, we demonstrate the deep collapse mode of CMUTs, which was introduced in [5]. In Fig. 12, we plotted the generated pressure under fixed amplitude $(100 \mathrm{~V})$ rising and falling voltage steps. Pressure generation of the same CMUT plate is depicted as a function of the gap under the plate. For gap heights larger than $420 \mathrm{~nm}$, the plate does not go into the collapsed state and a pressure of $0.25 \mathrm{MPa}$ is obtained. This part at the right-hand side of the figure demonstrates the conventional uncollapse mode of operation, in which the plate does not come in contact with the substrate. When the

\footnotetext{
${ }^{6} \mathrm{~A}$ movie depicting this artifact is uploaded as a supplementary material. 国
}

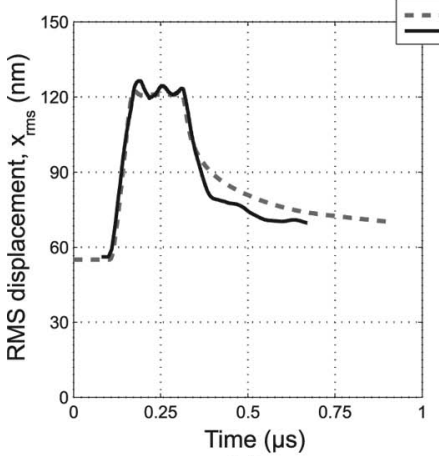

(a)

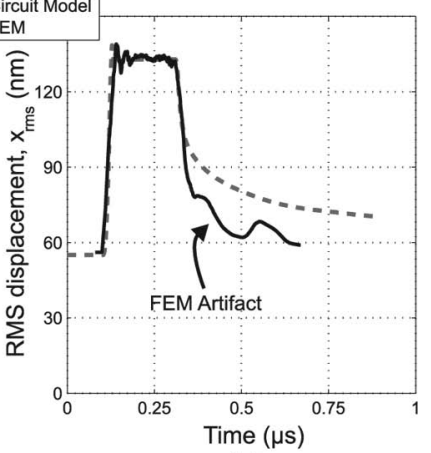

(b)
Fig. 11. Variation of $x_{\text {rms }}$ as calculated by FEM (solid lines) and equivalent circuit simulation (dashed lines) as a function of time, when the CMUT is excited by $10 \mathrm{~ns}$ rise or fall time pulses with (a) $100 \mathrm{~V}$ and (b) $200 \mathrm{~V}$ amplitudes. The simulated CMUT has a silicon nitride plate with $a=30 \mu \mathrm{m}, t_{m}=2 \mu \mathrm{m}, t_{i}=0.4 \mu \mathrm{m}, t_{g}=0.16 \mu \mathrm{m}$ resulting in a collapsed state under atmospheric pressure.

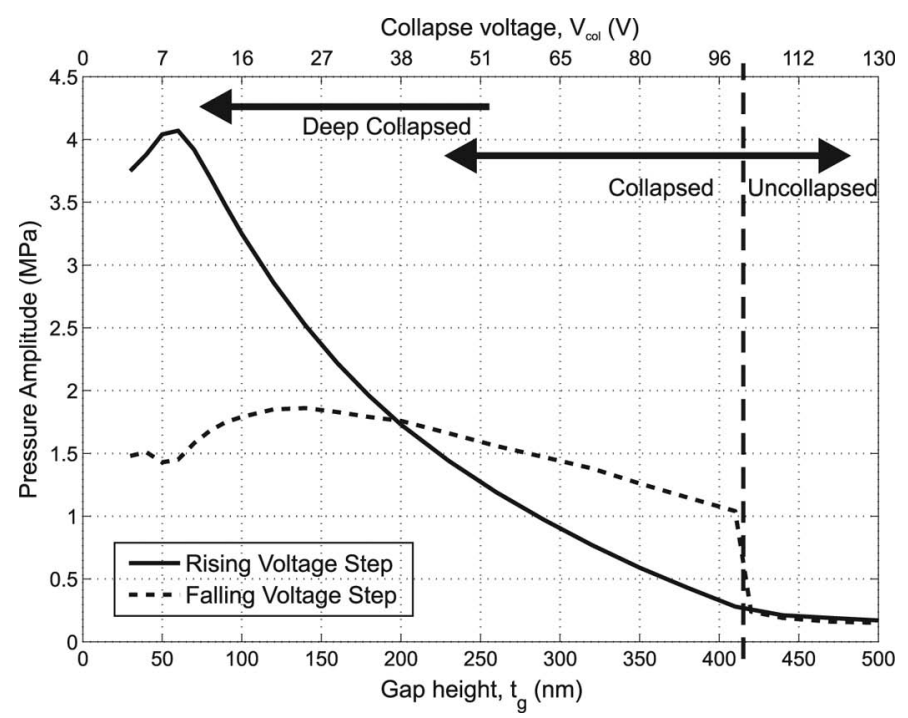

Fig. 12. Peak pressure output of a CMUT array with a step excitation as a function of the gap height as found from the electrical equivalent circuit. The input step has an amplitude of $100 \mathrm{~V}$ and $10 \mathrm{~ns}$ rise and fall times. Considered plates has $a=30 \mu \mathrm{m}, t_{m}=1.4 \mu \mathrm{m}$, and $t_{i}=0.4 \mu \mathrm{m}$ with varying $t_{g}$.

gap height is small enough to make the collapse voltage less than $100 \mathrm{~V}$, the generated pressure by a falling voltage step suddenly increases. This is expected, because the amount of energy stored in a collapsed plate is larger than the energy stored in an uncollapsed plate. Stored energy is radiated to the medium as a pressure wave during the falling voltage step. During the rising voltage step, on the other hand, the electrostatic attraction force generated between the electrodes is very small at first and slowly increases. Therefore, the increase in the pressure generation during the rising voltage step is somewhat slower, as 


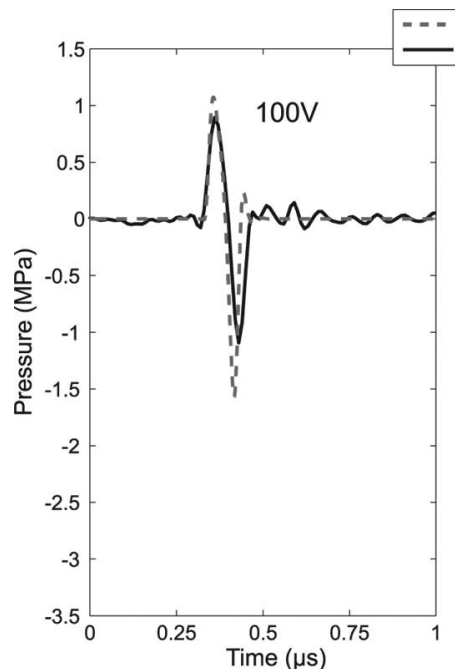

(a)

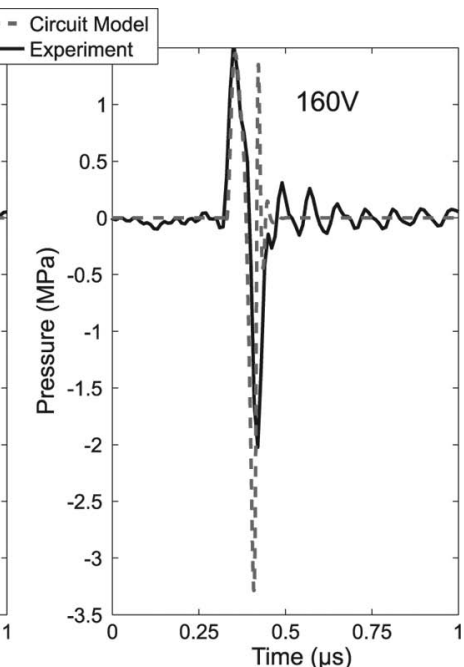

(b)
Fig. 13. Comparison of the equivalent circuit simulations (dashed lines) to experimental results (solid lines). The CMUTs used in the experiments have $a=30 \mu \mathrm{m}, t_{m}=1.4 \mu \mathrm{m}, t_{q}=0.2 \mu \mathrm{m}$, and $t_{i}=0.4 \mu \mathrm{m}$. They are excited with signals of 120 and $160 \mathrm{~V}$ negative amplitudes on equal amplitude dc biases.

seen in the figure. Nevertheless, if the separation of the electrodes is small (i.e., the gap and the collapse voltage are small) the peak pressure that is generated by the CMUT plate reaches a maximum. For example, at a gap height of about $60 \mathrm{~nm}$, a collapsing plate generates a pressure output of $4 \mathrm{MPa}$, demonstrating the attractiveness of the deep collapse mode. Note that with such a small gap height, CMUTs have a collapse voltage less than $10 \mathrm{~V}$.

\section{Comparison of the Equivalent Circuit Model to Experiment}

We compare the results of the equivalent circuit simulations to experimental results obtained from fabricated devices $^{7}$ in Fig. 13. We approximate the radiation resistance by (11), because $a \ll \lambda$ and $A \approx \lambda$ at $5 \mathrm{MHz}$. However, this assumption is valid for the uncollapsed-case CMUTs. Additionally, such radiation impedance requires the cells to be placed in a closely packed hexagonal network, where the fill ratio is $90.7 \%$. However, the tested array has 11 by 11 cells with $5 \mu \mathrm{m}$ spacing in a rectangular grid distribution, resulting in a $67 \%$ fill ratio, and the total aperture is $0.71 \times 0.71 \mathrm{~mm}$. Therefore, the generated peak-to-peak pressure output is measured to be smaller when compared with circuit simulations. This difference can be attributed to the difference in the fill ratios.

We can identify a ringing at the end of the experimental acoustic pulse. The ringing, which is at $12.2 \mathrm{MHz}$, is due to the thickness ringing of the substrate. Although the positive cycles of the output pressure waves are well predicted by the equivalent circuit simulations, the negative

\footnotetext{
${ }^{7}$ The silicon nitride fabrication process of the array is performed on a $\sim 340 \mu \mathrm{m}$ thick silicon substrate, similar to that in [23] and as discussed in [5]. The material parameters of the plates are given in Table II.
}

cycles are not. The difference may be attributed to the frequency-independent radiation resistance assumption.

To achieve a higher accuracy for predicting the response of a complete array, a more accurate radiation impedance model is needed. First, the radiation impedance of a single collapsed plate as a function of the contact radius or lumped displacement measure must be determined. The resulting radiation impedance of single cell must be used for the calculation of the mutual impedances between two collapsed plates. Finally, the radiation impedance of a single plate and the mutual radiation impedances must be combined to achieve the radiation impedance of a complete array of CMUT plate. This procedure was applied to uncollapsed mode CMUTs in [21] and can be used to calculate the radiation impedance seen by CMUT arrays of any size.

\section{Conclusions}

In this work, we have developed a parametric equivalent circuit model for CMUTs. Exact analytical equations are used to determine the relation between the deflection of a CMUT plate and the forces acting on the plate. In the static case, the balancing forces are the force due to atmospheric pressure, the electrical attraction force, and the opposing restoring force of the plate. The equivalent circuit includes the effects of plate mass and the loading of the immersion medium. The simulation of the equivalent circuit can be completed much faster than FEM simulations, but the results are within $3 \%$ of each other in amplitude and $6 \%$ of each other in frequency. With an arbitrary excitation of CMUT, one can deduce the time waveform of the acoustic signal as well as the instantaneous position of the plate. The proposed equivalent circuit model is a very strong design and simulation tool for collapse- and/or uncollapsed-mode CMUTs. We note that the accuracy of the equivalent circuit can be improved by adding a more exact radiation impedance model.

\section{ApPEndix A Correction FACTOR, $\kappa$}

An equivalent circuit model of a distributed system, such as a CMUT plate, requires a consistent use of lumped variables (through and across variables). Moreover, the selection of the lumped displacement measure should not affect the kinetic and potential energies of the plate. The kinetic energy, $E_{\mathrm{k}}$, of a CMUT plate is calculated as

$$
\begin{aligned}
E_{k}(t) & =\int_{0}^{a} \frac{1}{2}\left(\rho t_{m} 2 \pi r \mathrm{~d} r\right) v^{2}(r, t) \\
& =\frac{1}{2} \rho t_{m} \pi a^{2}\left[\frac{1}{\pi a^{2}} \int_{0}^{a} 2 \pi r v^{2}(r, t) \mathrm{d} r\right] \\
& =\frac{1}{2} m v_{\mathrm{rms}}^{2}(t) .
\end{aligned}
$$


This result shows that choosing the rms value as the lumped variable gives the correct kinetic energy of a plate. On the other hand, the stored potential energy, $E_{\mathrm{p}}$ in a deflected plate by a force, $F$ caused by a uniform pressure, $P$, is calculated by evaluating the following integral:

$$
\begin{aligned}
E_{p}(t) & =\int_{0}^{a} x(r, t) P 2 \pi r \mathrm{~d} r \\
& =P \pi a^{2}\left[\frac{1}{\pi a^{2}} \int_{0}^{a} 2 \pi r x(r, t) \mathrm{d} r\right] \\
& =F x_{\mathrm{avg}}(t)=\left(F \frac{x_{\mathrm{avg}}(t)}{x_{\mathrm{rms}}(t)}\right) x_{\mathrm{rms}}(t),
\end{aligned}
$$

where the expression in the brackets is equal to $x_{\text {avg. }}$. If $x_{\text {avg }}$ is chosen as the lumped measure, the Fx product represents the potential energy correctly. Because we use $x_{\text {rms }}$ rather than $x_{\text {avg }}$ as the lumped variable, $F$ needs to be divided by $\kappa=x_{\text {rms }} / x_{\text {avg }}$.

\section{Appendix B}

\section{Static Deflection of a Clamped Circular Plate}

\section{A. Uncollapsed State}

The expression for the deflection, $x(r)$, of a clamped circular plate under uniform pressure, $P$, was derived by Timoshenko [18, p. 55]:

$$
x(r)=\frac{P}{64 D}\left(a^{2}-r^{2}\right)^{2} .
$$

Here, $D$ is the flexural rigidity of the plate given by $E t_{m}^{3} /\left(12\left(1-\nu^{2}\right)\right)$, where $E$ is the Young's modulus and $\nu$ is the Poisson's ratio. The average displacement of the plate can be written as given in [14]:

$$
x_{\text {avg }}=\frac{P a^{4}}{192 D} .
$$

Because $\kappa=x_{\mathrm{rms}} / x_{\text {avg }}=3 / \sqrt{5}$ in the uncollapsed state [15], we can write

$$
x_{\mathrm{rms}}=\frac{P a^{4} \kappa}{192 D}=\frac{3 a^{2}}{320 \pi D} F_{\mathrm{r}},
$$

where $F_{\mathrm{r}}$ is the lumped restoring force of the plate, which is corrected with respect to the rms through the variable.

The capacitance, $C$, of a deflected CMUT in the uncollapsed state is given as

$$
C=\frac{\varepsilon_{0} \pi a^{2} \operatorname{atanh}\left(\sqrt{\frac{\sqrt{5} x_{\mathrm{rms}}}{t_{g^{\prime}}}}\right)}{\sqrt{\sqrt{5} t_{g^{\prime}} x_{\mathrm{rms}}}},
$$

where $t_{g^{\prime}}=t_{g}+t_{i} / \varepsilon_{\mathrm{r}}$, with $\varepsilon_{\mathrm{r}}$ being the relative permittivity of the insulation material. The derivative of the capacitance with respect to $x_{\mathrm{rms}}$ is given in [14] as

$$
\frac{\mathrm{d} C}{\mathrm{~d} x_{\mathrm{rms}}}=\frac{\varepsilon_{0} \pi a^{2}}{2 t_{g^{\prime}} \frac{\sqrt{5}}{3} x_{\mathrm{rms}}\left(1-\frac{\sqrt{5} x_{\mathrm{rms}}}{t_{g^{\prime}}}\right)}-\frac{C}{\frac{2 \sqrt{5}}{3} x_{\mathrm{rms}}} .
$$

\section{B. Collapsed State}

The general solution for the deflection of a uniformly loaded circular plate derived by Timoshenko $[18$, p. 309] is used as the starting point:

$$
\begin{array}{r}
x(r)=C_{1}+C_{2} \ln r+C_{3} r^{2}+C_{4} r^{2} \ln r+\frac{r^{4}}{64 D} P, \\
\text { for } b \leq r \leq a .
\end{array}
$$

The boundary conditions for a plate as shown in Fig. 1 are

$$
\begin{gathered}
x(a)=0, \quad x(b)=t_{g} \\
\left.\frac{\mathrm{d} x(r)}{\mathrm{d} r}\right|_{r=a}=0,\left.\quad \frac{\mathrm{d} x(r)}{\mathrm{d} r}\right|_{r=b}=0 \\
M_{\mathrm{r}}(b)=-\left.D\left(\frac{\mathrm{d}^{2} x(r)}{\mathrm{d} r^{2}}+\frac{\nu}{r} \frac{\mathrm{d} x(r)}{\mathrm{d} r}\right)\right|_{r=b}=0,
\end{gathered}
$$

where $M_{\mathrm{r}}$ is the radial bending moment acting on the plate; $b$ is determined by solving (22) in terms of the four unknown constants of (19), which in turn are determined using the four boundary conditions of (20) and (21).

\section{Appendix C FEM Simulations}

The finite element simulations are done using ANSYS FEM Package (v12.1, Ansys Inc., Canonsburg, PA). A 2-D axisymmetric model of CMUT [5] is utilized. 8-node structural solid (PLANE82) elements are combined with electromechanical transducer elements (TRANS126) under the bottom surface nodes of the plate. A gap value (GAP) and a minimum gap value (GAPMIN) are needed to generate ground plane nodes and determine maximum possible deflection before contact. A contact stiffness factor, $\mathrm{FKN}=1$ is used to overcome convergence problems with a reasonable penetration at contact interface.

TRANS126 elements do not accept an insulating layer between the electrodes as in the case of CMUTs. Therefore, GAP and GAPMIN parameters are modified to perform an accurate simulation. The modified parameters are defined as

$$
\mathrm{GAP}=t_{g^{\prime}}=t_{g}+\frac{t_{i}}{\varepsilon_{\mathrm{r}}}, \quad \text { GAPMIN }=\frac{t_{i}}{\varepsilon_{\mathrm{r}}} .
$$

A fluid loading is included in the model for simulating the dynamic behavior of the CMUTs. A fluid column of $2 \mathrm{~mm}$ height is created over the plate using 2-D axisymmetric harmonic acoustic fluid (FLUID29) elements. The 
fluid-structure flags are enabled for coupling the structural motion as an acoustic wave in the medium. To ensure the dc stability, the transient effects are turned off in the first step and turned on at the rest of the simulation. The total duration of the simulation is fixed to $1 \mu \mathrm{s}$ and average pressure is recorded at $1 \mathrm{~mm}$ above the plate surface.

\section{REFERENCES}

[1] I. Ladabaum, X. Jin, H. T. Soh, A. Atalar, and B. T. Khuri-Yakub, "Surface micromachined capacitive ultrasonic transducers," IEEE Trans. Ultrason. Ferroelectr. Freq. Control, vol. 45, no. 3, pp. 678 690, 1998.

[2] R. O. Guldiken, J. Zahorian, F. Y. Yamaner, and F. L. Degertekin, "Dual-electrode CMUT with non-uniform membranes for high electromechanical coupling coefficient and high bandwidth operation," IEEE Trans. Ultrason. Ferroelectr. Freq. Control, vol. 56, no. 6, pp. 1270-1276, 2009.

[3] Ö. Oralkan, B. Bayram, G. G. Yaralioglu, A. S. Ergun, M. Kupnik, D. T. Yeh, I. O. Wygant, and B. T. Khuri-Yakub, "Experimental characterization of collapse-mode CMUT operation," IEEE Trans. Ultrason. Ferroelectr. Freq. Control, vol. 53, no. 8, pp. 1513-1523, 2006.

[4] Y. Huang, E. Hæggstrom, B. Bayram, X. Zhuang, A. S. Ergun, C.-H. Cheng, and B. T. Khuri-Yakub, "Comparison of conventional and collapsed region operation of capacitive micromachined ultrasonic transducers," IEEE Trans. Ultrason. Ferroelectr. Freq. Control, vol. 53, no. 10, pp. 1918-1932, 2006.

[5] S. Olcum, F. Y. Yamaner, A. Bozkurt, H. Köymen, and A. Atalar, "Deep collapse operation of capacitive micromachined ultrasonic transducers," 2010. submitted for publication.

[6] A. Bozkurt, F. L. Degertekin, A. Atalar, and B. T. Khuri-Yakub, "Analytic modelling of loss and cross-coupling in capacitive micromachined ultrasonic transducers," in Proc. IEEE Ultrasonics Symp., 1998, pp. 1025-1028.

[7] G. G. Yaralioglu, A. S. Ergun, and B. T. Khuri-Yakub, "Finite-element analysis of capacitive micromachined ultrasonic transducers," IEEE Trans. Ultrason. Ferroelectr. Freq. Control, vol. 52, no. 12, pp. 2185-2198, 2005.

[8] A. Lohfink and P. C. Eccardt, "Linear and nonlinear equivalent circuit modeling of CMUTs," IEEE Trans. Ultrason. Ferroelectr. Freq. Control, vol. 52, no. 12, pp. 2163-2172, 2005.

[9] A. Caronti, G. Caliano, A. Iula, and M. Pappalardo, "An accurate model for capacitive micromachined ultrasonic transducers," IEEE Trans. Ultrason. Ferroelectr. Freq. Control, vol. 49, no. 2, pp. 159-167, 2002.

[10] S. Olcum, M. N. Senlik, and A. Atalar, "Optimization of the gainbandwidth product of capacitive micromachined ultrasonic transducers," IEEE Trans. Ultrason. Ferroelectr. Freq. Control, vol. 52, no. 12 , pp. 2211-2219, 2005.

[11] H. Köymen, M. N. Senlik, A. Atalar, and S. Olcum, "Parametric linear modeling of circular CMUT membranes in vacuum," IEEE Trans. Ultrason. Ferroelectr. Freq. Control, vol. 54, no. 6, pp. 1229$1239,2007$.

[12] A. Caronti, R. Carotenuto, and M. Pappalardo, "Electromechical coupling factor of capacitive micromachined ultrasonic transducers," J. Acoust. Soc. Am., vol. 113, no. 1, pp. 279-288, 2003.

[13] W. P. Mason, Electromechanical Transducers and Wave Filters. New York, NY: Van Nostrand, 1942.

[14] I. O. Wygant, M. Kupnik, and B. T. Khuri-Yakub, "Analytically calculating membrane displacement and the equivalent circuit model of a circular CMUT cell," in Proc. IEEE Ultrasonics Symp., 2008, pp. 2111-2114.

[15] H. K. Oguz, S. Olcum, M. N. Senlik, V. Tas, A. Atalar, and H. Köymen, "Nonlinear modeling of an immersed transmitting capacitive micromachined ultrasonic transducer for harmonic balance analysis," IEEE Trans. Ultrason. Ferroelectr. Freq. Control, vol. 57, no. 2, pp. $438-447,2010$.

[16] B. Bayram, Ö. Oralkan, A. S. Ergun, E. Hæggström, G. G. Yaralioglu, and B. T. Khuri-Yakub, "Capacitive micromachined ultrasonic transducer design for high power transmission," IEEE Trans. Ultrason. Ferroelectr. Freq. Control, vol. 52, no. 2, pp. 326-339, 2005.
[17] B. Bayram, G. G. Yaralioglu, M. Kupnik, A. S. Ergun, Ö. Oralkan, A. Nikoozadeh, and B. T. Khuri-Yakub, "Dynamic analysis of capacitive micromachined ultrasonic transducers," IEEE Trans. Ultrason. Ferroelectr. Freq. Control, vol. 52, no. 12, pp. 2270-2275, 2005.

[18] S. Timoshenko and S. W. Woinowsky-Krieger, Theory of Plates and Shells, 2nd ed. New York, NY: McGraw Hill, 1959.

[19] E. Ventsel and T. Krauthammer, Thin Plates and Shells, 1st ed. New York, NY: Marcel Dekker, 2001.

[20] M. Kupnik, I. O. Wygant, and B. T. Khuri-Yakub, "Finite element analysis of stress stiffening effects in CMUTs," in Proc. IEEE Ultrasonics Symp., 2008, pp. 487-490.

[21] M. N. Senlik, S. Olcum, H. Köymen, and A. Atalar, "Radiation impedance of an array of circular capacitive micromachined ultrasonic transducers," IEEE Trans. Ultrason. Ferroelectr. Freq. Control, vol. 57, no. 4, pp. 969-976, 2010.

[22] P. R. Stepanishen, "Impulse response and radiation impedance of an annular piston," J. Acoust. Soc. Am., vol. 56, no. 2, pp. 305-312, 1974.

[23] J. Knight, J. McLean, and F. L. Degertekin, "Low temperature fabrication of immersion capacitive micromachined ultrasonic transducers on silicon and dielectric substrates," IEEE Trans. Ultrason. Ferroelectr. Freq. Control, vol. 51, no. 10, pp. 1324-1333, 2004.

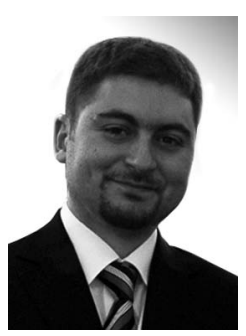

Selim Olçum was born in Chicago, IL, in 1981. He received his B.S., M.S., and Ph.D. degrees in electrical engineering from Bilkent University, Ankara, Turkey, in 2003, 2005, and 2010, respectively. He worked as a guest researcher at the National Institute of Standards and Technology, Semiconductor Electronics Division, Gaithersburg, MD, during the summers of 2002 and 2003. He was a visiting scholar in the Micromachined Sensors and Transducers Laboratory of the Georgia Institute of Technology, Atlanta, GA, in 2006. He is currently an instructor in the Electrical and Electronics Engineering Department at Bilkent University. His current research interests include micromachined sensors and actuators, transducer design and modeling, and plasmonic sensors.

Dr. Olçum was a fellow of ASELSAN during his Ph.D. study.

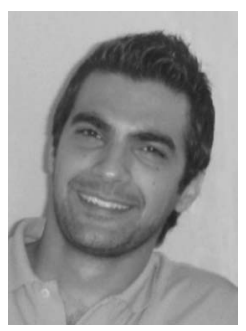

Yalçn Yamaner received his B.Sc. degree from Ege University, Izmir, Turkey, in 2003 and his M.Sc. degree from Sabanc University, Istanbul, Turkey, in 2005, all in electrical and electronics engineering. He worked as a visiting researcher at VLSI Design and Education Center (VDEC), during the summer of 2006. He was a visiting scholar in the Micromachined Sensors and Transducers Laboratory of the Georgia Institute of Technology, Atlanta, GA, in 2008. He is currently working toward his Ph.D. degree in electronics engineering at Sabanc University. His current research interests include micromachined sensors, transducer modeling, and fabrication.

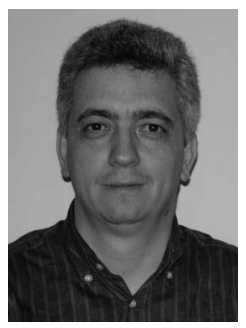

Ayhan Bozkurt received his B.Sc., M.Sc., and Ph.D. degrees from Bilkent University, Ankara, Turkey, in 1992, 1994, and 2000, respectively, all in electrical and electronics engineering. He is currently working as an Associate Professor in the Electronics Engineering Program of the Faculty of Engineering and Natural Sciences of Sabanc University, Istanbul, Turkey. His research interests are transducer modeling and fabrication and frontend integrated circuit design. 


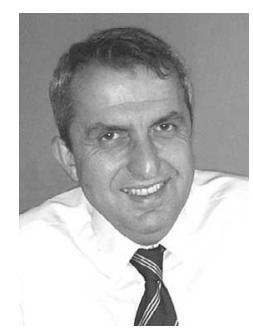

Hayrettin Köymen received the B.Sc. and M. Sc. degrees from the Middle East Technical University (METU), Ankara, Turkey in 1973 and 1976, respectively, and the Ph.D. degree from Birmingham University, UK, in 1979, all in electrical engineering. He worked as a faculty member in the Marine Sciences Dept. (Mersin) and Electrical Engineering Dept. (Ankara) of METU, from 1979 to 1990, and at Bilkent University since 1990, where he is a professor. His research activities have included underwater acoustic and ultrasonic transducer design, acoustic microscopy, ultrasonic NDT, biomedical instrumentation, mobile communications, and spectrum management.

Prof. Köymen is a fellow of IET (formerly IEE).

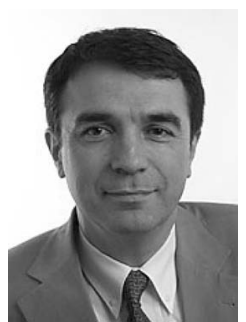

Abdullah Atalar received a B.S. degree from the Middle East Technical University, Ankara, Turkey, in 1974, and M.S. and Ph.D. degrees from Stanford University, Stanford, CA, in 1976 and 1978, respectively, all in electrical engineering. He worked in Hewlett-Packard Labs, Palo Alto, CA in 1979. From 1980 to 1986 , he was on the faculty of the Middle East Technical University as an Assistant Professor. In 1986, he joined Bilkent University as the chairman of the Electrical and Electronics Engineering Department and served in the founding of the Department where he is currently a Professor. In 1995, he was a Visiting Professor of Stanford University. From 1996 to 2010, he was the Provost of Bilkent University. He is presently the Rector of the same university. His current research interests include micromachined devices and microwave electronics.

Prof. Atalar was awarded the Science Award of TUBITAK in 1994. He is a Fellow of IEEE and a member of the Turkish Academy of Sciences. 